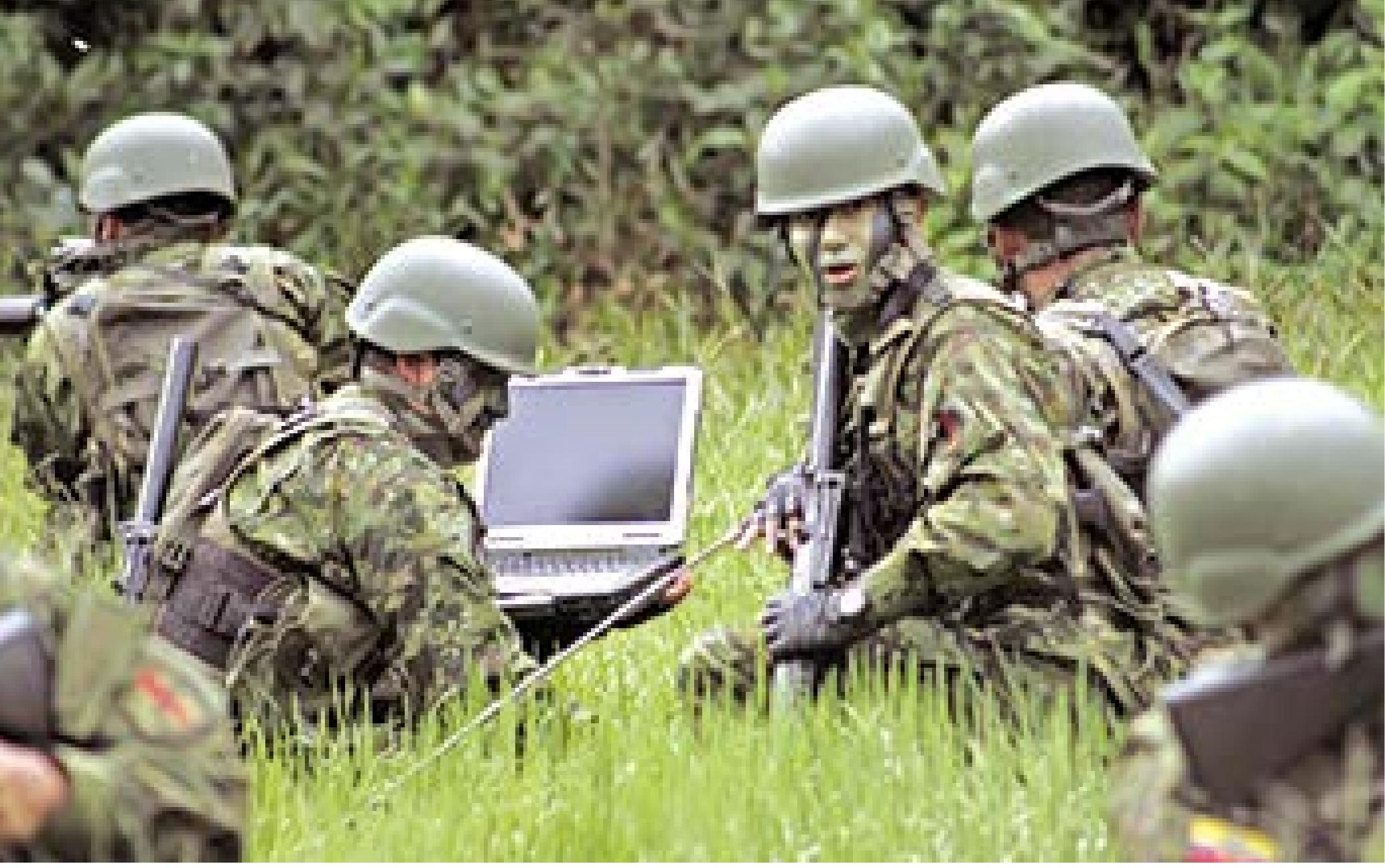

\title{
LA VISIÓN POR COMPUTADORA Y LAS FUTURAS APLICACIONES TECNOLÓGICAS EN DIVERSOS ESCENARIOS
}

\section{RESUMEN}

Mayo. de Com. Henry Cruz Carrillo

El avance tecnológico avanza con pasos agigantados que permite perfeccionar procedimientos y actividades en la vida cotidiana, y que deben ser de mucha utilidad en el sector de la defensa. Este desarrollo tecnológico ha permitido solventar las necesidades fisiológicas, mediante el uso de plataformas inteligentes e incluso con el desarrollo de inteligencia artificial. En el campo de seguridad se hace impredecible el uso de estas bondades por medio de la visión por computadora, que permitirá un monitoreo y protección del medio ambiente así como en el apoyo a las operaciones en la seguridad y defensa.

Palabras claves Inteligencia artificial, monitoreo, sensores, dispositivos, tecnología

\section{ABSTRACT}

Technological advancement is advancing with leaps and bounds that allow perfecting procedures and activities in daily life, and that should be very useful in the defense sector. This technological development has made it possible to solve physiological needs, through the use of intelligent platforms and even with the development of artificial intelligence. In the field of security, the use of these benefits through computer vision becomes unpredictable, which will allow monitoring and protection of the environment as well as in support of security and defense operations.

Keywords: Artificial intelligence, monitoring, sensors, devices, technology 


\section{Introducción}

La supervivencia de la humanidad está ligada al desarrollo de la tecnología, la misma que le ha permitido solventar necesidades fisiológicas, de seguridad, sociales, entre otras. Según el Hiperciclo de Gartner ${ }^{1}$, las principales tecnologías que se encuentran en el pico de de la innovación y la expectación, están asociadas a la inteligencia artificial $^{2}$, el aprendizaje automático ${ }^{3}$ y las plataformas del internet de las cosas (IoT) ${ }^{4}$ (Panetta, 2018). Justamente, a partir de estas tecnologías se espera que para los próximos años se desarrollen un sin número de aplicaciones relacionadas a la toma de decisiones en; el transporte (vehículos autónomos), asistentes personales y grupales (robots autónomos inteligentes, asistentes virtuales), hogares digitales conectados, interfaces cerebro - computadora, biochips, etc.

Aunque pareciera que estas tecnologías proceden de la ciencia ficción, no es así, pues en este momento grupos y redes de investigación alrededor del mundo están trabajando en perfeccionar las mismas, de tal forma de disponer de insumos tecnológicos innovadores. Muchas de las aplicaciones en desarrollo, dependen de sensores que proporcionen información del entorno $y$ que permiten al organismo artificial ${ }^{5}$ entrenar una serie de datos, para la posterior toma de decisiones. Precisamente la visión por computadora o también llamada visión artificial es una rama de la inteligencia artificial, que facilita a este organismo interpretar el entorno que le rodea a través del procesamiento digital de las imágenes y/o señales.

El incremento de las capacidades de los procesadores, redes de datos y telecomunicaciones, así como de los sistemas de información han permitido reducir los tiempos de cálculo y retardo, consiguiendo que el tratamiento de la información visual sea en tiempo real. Es necesario precisar también que, uno de los principales sentidos del ser humano es la vista, y la visión artificial busca homologar el funcionamiento visual humano a los sistemas autónomos inteligentes, adquiriendo y procesando la información del entorno a través de diferentes tipos de sensores y procesadores computarizados, aprovechando las capacidades que en la actualidad se dispone.

\section{Los sensores ópticos y la visión computadora}

Los sensores ópticos son fuente casi infinita de información, la misma que se constituye en insumo para uso y aplicación en la visión por computadora. Estos sensores los podemos encontrar en los sistemas de video vigilancia de hogares, ciudades, transporte, teléfonos inteligentes, sistemas de vigilancia y teledetección satelital, etc. Las capacidades de estos sistemas se incrementan considerablemente al estar interconectados a las redes de datos e internet, dándoles un carácter global.

La información que proporcionan los sensores ópticos es analizada de tal forma que sea útil en la detección, seguimiento y reconocimiento de un objetivo en específico o múltiples objetivos que sean de interés, esto es utilizado, por ejemplo, en la conducción autónoma para; interpretar el entorno, determinar distancias, reconocer luces semafóricas, detectar pasos peatonales, reconocer y discriminar peatones de coches así como para actuar como asistentes en la conducción en condiciones de visibilidad y clima adversos (Cruz, 2016).

Por otra parte, la implementación de sistemas de video vigilancia que sean inteligentes, permite aportar en el desarrollo de ciudades también inteligentes, debido a que los centros de monitoreo remoto dejan de ser pasivos y se convierten en activos, ya que la inteligencia artificial y la visión por ordenador, los convierten en centros de toma de decisiones autónomas, generación automática de información y alarmas ante eventos inusuales, anómalos, de respuesta rápida o de emergencia. De esta manera los algoritmos de visión por computadora son capaces de discernir e identificar cuando ocurren atascos vehiculares, tráfico denso, choques vehiculares, aglomeraciones de gente, disturbios en lugares públicos (calles, estadios, plazas, etc.) así como permiten la identificación de presuntos quebrantadores de la ley y el orden público (Cruz Carrillo, 2017).

La visión por computadora también aporta al monitoreo y protección del medio ambiente así como ayuda en la gestión de emergencias naturales o antrópicas, por ejemplo los algoritmos desarrollados son capaces de detectar el fuego forestal e incendios en áreas urbanas, tanto para plataformas de video vigilancia fijas, a través de torres o para sistemas de

\footnotetext{
1 Hiperciclo de Gartner curva gráfica y estadística de la madurez, adopción, proyección y aplicación de una tecnología en específico.

2 Inteligencia Artificial (Artificial Intelligence) es la capacidad de razonamiento e interpretación que muestran las máquinas computacionales.

3 Aprendizaje Automático (Machine Learning), rama de la inteligencia artificial, cuyo objetivo es desarrollar técnicas que permitan que las computadoras aprendan.

4 Internet de las Cosas (Internet of Things-IoT) se refiere a una interconexión digital de objetos cotidianos con internet y que les permite interactuar entre ellos.

5 Organismo artificial, representa una máquina computacional que dispone de inteligencia artificial (humanoides, supercomputadoras, drones, etc.)
} 
video vigilancia móviles a través de los vehículos aéreos no tripulados (UAV's) ${ }^{6}$ (Cruz H. E., 2016). Así mismo permiten detectar la emisión de flujos piro-plásticos, gases, vapor de agua y cenizas durante una erupción volcánica así como inundaciones o desbordamiento de ríos. Por otra parte, también son útiles para la detección

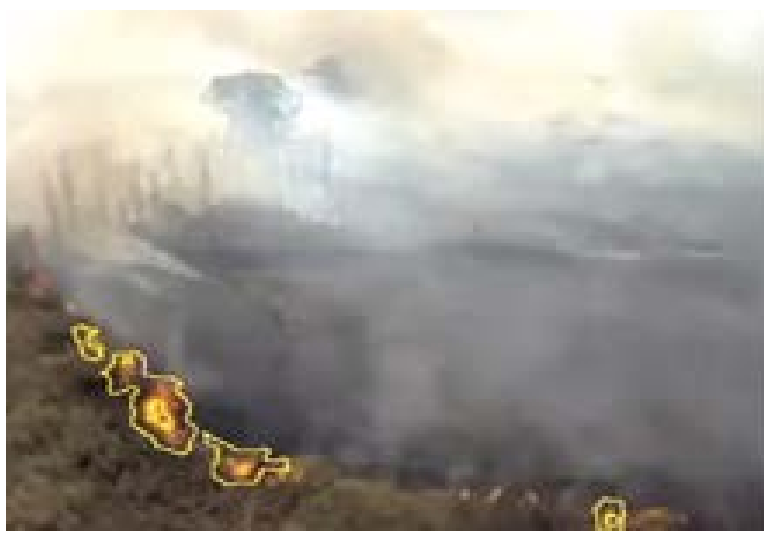

Figura. 1. (a) Detección automática de incendios forestales. Sensors, FFDI, Henry Cruz, Martina Eckert, Juan Meneses 2016 de deforestación de bosques densos y selváticos (Cruz H. O., 2017), así como para realizar estimaciones de densidad de los bosques. En la Fig.1, se observa la detección automática de incendios forestales y zonas deforestadas, información que es registrada a través del empleo de UAV's.

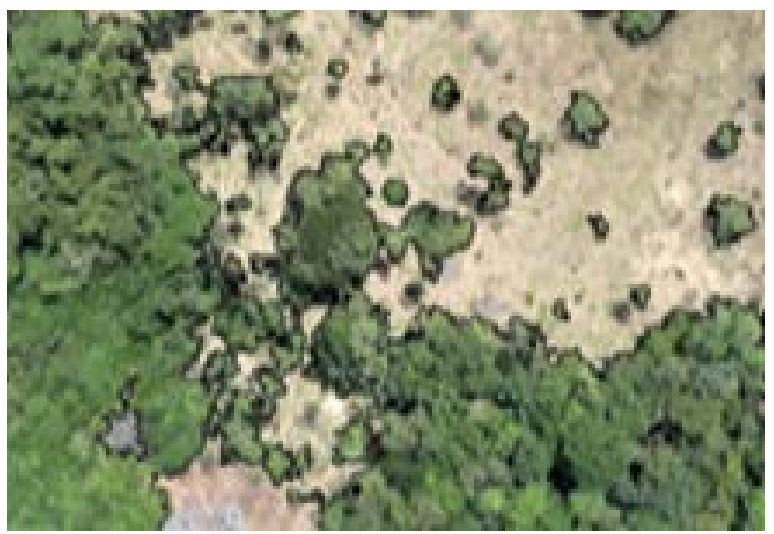

Figura. 1. (b) Detección automática de zonas deforestas. IEEE-TGRS Henry Cruz, Martina Eckert, Juan Meneses 2017.
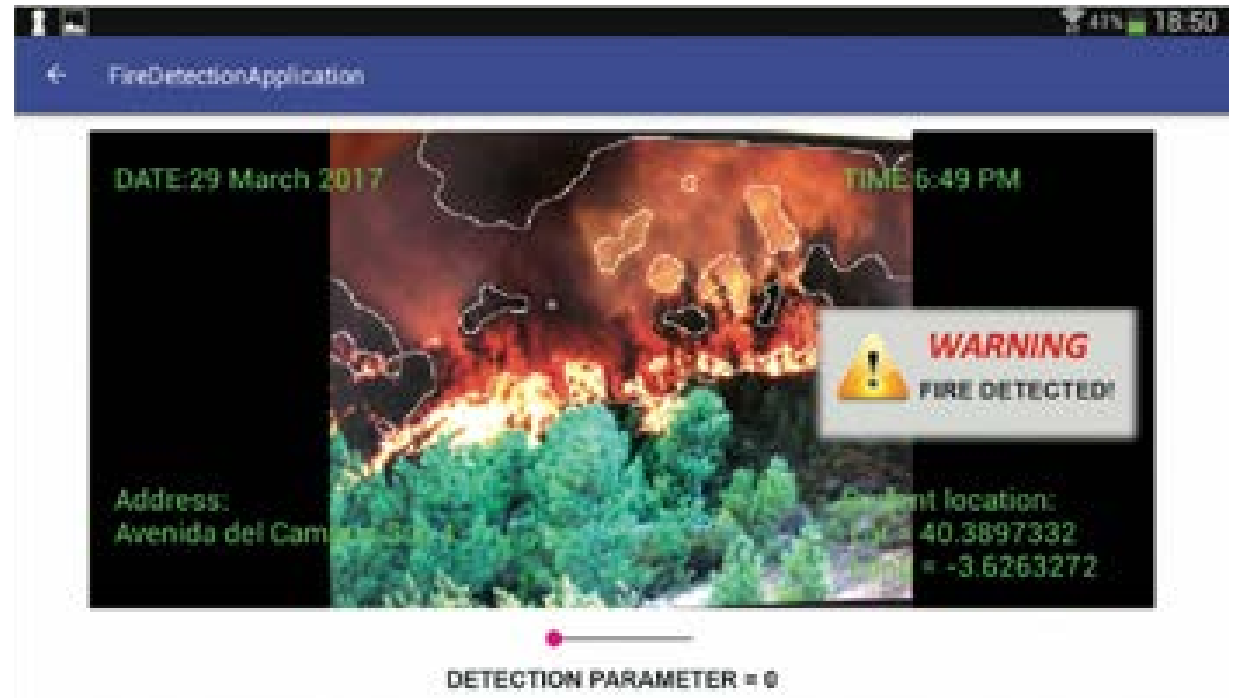

Figura 2. Generación de Realidad Aumentada (localización, fecha, hora, segmentación, alarma) de un fuego forestal mostrada por una tableta electrónica. (Tomada de Cruz Carrillo, H. O. 2017. Contribución a la detección de objetivos específicos para aplicaciones de vigilancia con realidad aumentada, ETSIST)

Existen otras aplicaciones asociadas al desarrollo de la agricultura y manejo de granjas autónomas, control de calidad en la agroindustria e industria farmacéutica, análisis de fisuras (represas, centrales nucleares, infraestructuras sensibles en general), análisis de resistencia de materiales, análisis y clasificación de especies biológicas, análisis y detección de tumoraciones o zonas con daños en órganos humanos, análisis e interpretación del comportamiento humano, reconocimiento facial, etc.

Un aspecto interesante es, la contribución de la visión por computadora al desarrollo de la realidad aumentada (RA). La RA representa la capacidad que tienen los dispositivos inteligentes (teléfonos, tabletas, asistentes personales, robots, etc.) de proporcionar información virtual de un objetivo y/o situación, en tiempo real (Fig. 2). El procesamiento digital de las imágenes a través de diversos algoritmos computacionales proporciona información intrínseca del objeto u objetivo, siendo útil para establecer las condiciones físicas del mismo (tamaños, localización, dimensiones, composición, características, etc.) o determinar factores externos que le afectan (presión, temperatura, radiación, situación, etc.) (Cruz Carrillo, 2017).

6 UAV's (Unnamed Aerial Vehicle) Vehículo Aéreo No Tripulado 


\section{Visión por computadora en la defensa}

En las décadas anteriores, el concepto de guerra, el comando y control en el campo de batalla, no conciben la automatización como una forma esencial de generar ventajas y aprovecharlas en el combate. Hoy en día el panorama luce diferente ya que la inteligencia artificial, la visión por computadora, la realidad aumentada, proporcionan capacidades de acción e información que son elementos claves en la toma de decisiones, ejemplos de esto se puede observar en conflictos y guerras post contemporáneas como la Segunda Guerra del Golfo y la guerra contra el estado islámico, en donde los UAV's son capaces de interpretar, definir y fijar objetivos estrictamente militares, separándolos de los que no lo son, evitando de esta forma daños colaterales en especial a la población civil, las llamadas bombas inteligentes son una muestra de ello.

Los sistemas UAV, UUV (vehículos subacuáticos no tripulados), USV (vehículos de superficie no tripulados) y los UGV (vehículos terrestres no tripulados), llevan a cabo actividades tales como inteligencia, vigilancia, adquisición de objetivos y reconocimiento (ISTAR), estos equipos y sistemas se utilizan para obtener información en tiempo real, así como pueden constituirse en vehículos autónomos de combate. La combinación de inteligencia artificial

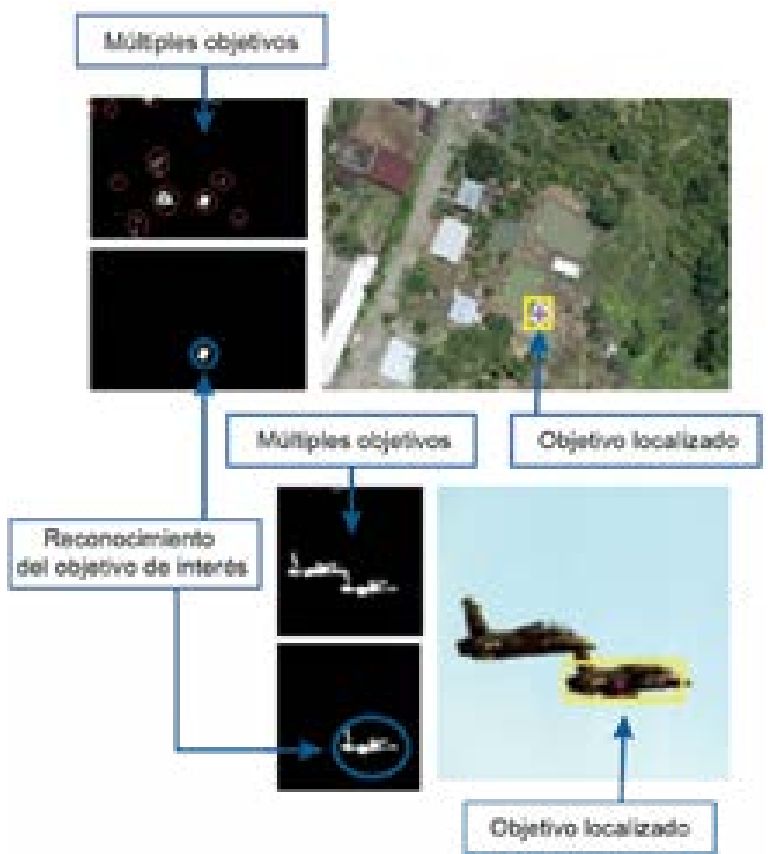

Figura 3. Reconocimiento y localización de un objetivo específico entre múltiples objetivos (Tomado de A RealTime Method to Remotely Detect a Target based on Color Average and Deviation, Henry Cruz, Springer 2018) asociada a la información obtenida por los sensores precisamente, le proporciona capacidades intrínsecas únicas a estos sistemas y les permiten actuar con sorpresa, masa y flexibilidad. En la Fig. 3, se observa como un organismo inteligente puede reconocer y discriminar un objetivo en específico de entre otros objetivos y localizarlo a través de la visión por computadora.

Además, los organismos cibernéticos terrestres (robots humanoides), a través de la visión por computadora y la inteligencia artificial, son capaces de tomar decisiones autónomas en combate, pueden acompañar al combatiente en la batalla y realizar tareas de alto riesgo como: detonación de bombas, infiltraciones, rescate y evacuación, así como acciones de asalto y neutralización de objetivos específicos. Precisamente, las diferentes generaciones y modelos desarrollados por la compañía Norte Americana Boston Dynamics ${ }^{\circledR}$ y supervisada por la $\mathrm{DARPA}^{7}$, disponen de ciertas características que hacen prever la participación de nuevos actores en las guerras del futuro (Boston Dynamics, 2018). En la Fig. 4, se muestra el empleo de robots logísticos empleados en las operaciones militares del Ejército de los EE.UU. Lógicamente toda la información que se obtiene de los diferentes tipos de sensores, se transporta a través de redes avanzadas de telecomunicaciones y de datos con alta calidad (QoS) y disponibilidad de servicio. Todo hace pensar que en un futuro mediato, todas estas tecnologías y capacidades autónomas definitivamente cambiarán la concepción ISTAR.

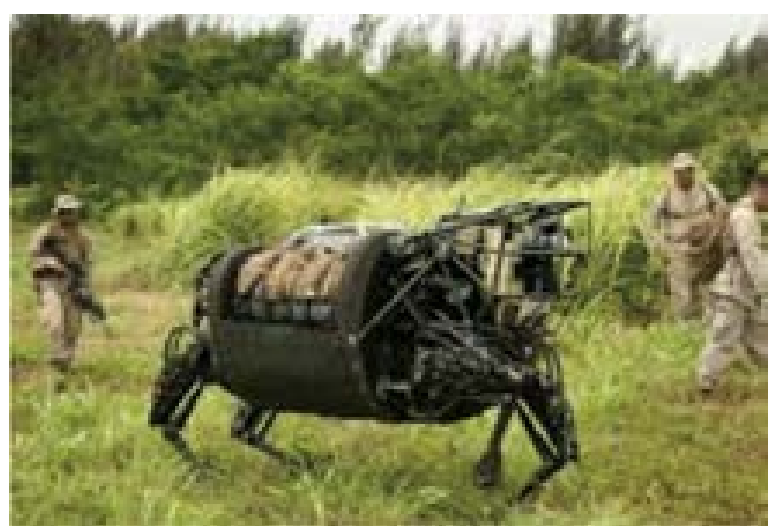

Figura 4. Robot empleado como abastecedor y elemento de transporte durante la ejecución de operaciones militares (Tomado de la pagina web https://www.bostondynamics. com/robots)

\footnotetext{
7 DARPA - Defense Advanced Research Projects Agency. División de desarrollo de nuevas tecnologías del Departamento de Defensa de los EE.UU.
} 


\section{Conclusiones}

La tecnología está en constante actualización, adaptación y evolución, el ser humano se ha servido de ésta, para solventar todo tipo de necesidades.

Las tendencias de implementación de nuevas tecnologías abarcan principalmente a campos asociados al desarrollo de la inteligencia artificial, así como de la visión artificial como una rama de ésta.

El incremento de la capacidad de procesamiento y transmisión de la información de los ordenadores, así como de las redes de datos ha permitido que las aplicaciones basadas en el procesamiento digital de imágenes y visión por computadores sean en la actualidad ejecutadas en tiempo real.
La visión por computadora se aplica en diversos campos como son: la seguridad y defensa, gestión de riesgos, control y monitoreo ambiental, transporte, administración de ciudades inteligentes, industria agro alimentaria, farmacéutica, medicina, construcción, etc.

La visión por computadora y la inteligencia artificial definitivamente cambiarán la dinámica del campo de batalla, ya que los organismos autónomos serán capaces de ejecutar misiones de todo tipo y acompañarán a los combatientes en el desarrollo de las acciones bélicas, sin lugar a duda en el futuro mediato se avizora una nueva forma de hacer guerra y solventar los conflictos armados.

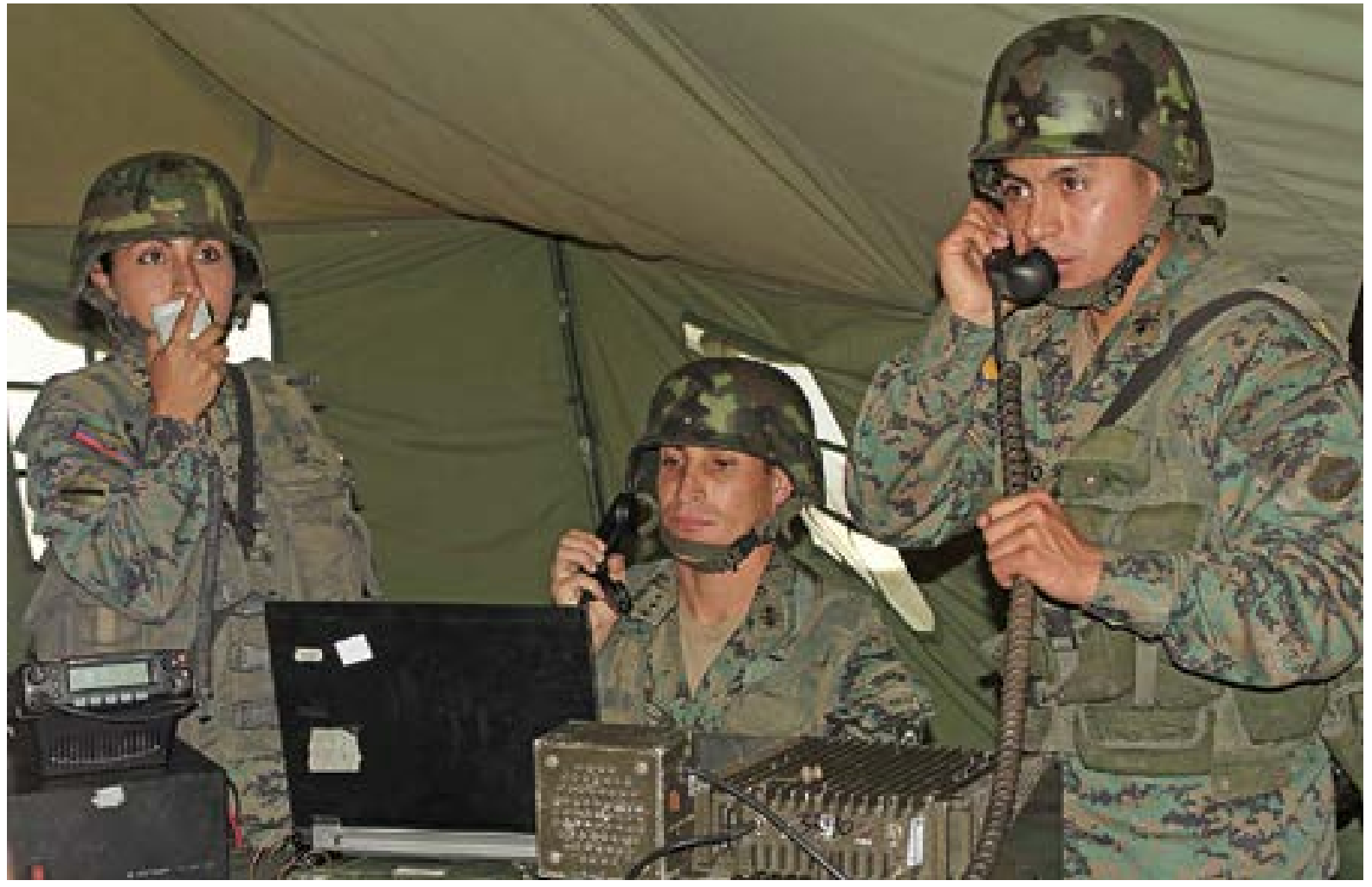

Personal militar realizando pruebas con el equipo de comunicaciones.

Fuente: Archivo fotográfico OPSIC - Ejército Ecuatoriano

[1] Boston Dynamics. (Enero de 2018). bostondynamics. Recuperado el 25 de 09 de 2018 , de https://www. bostondynamics.com

[2] Cruz Carrillo, H. O. (24 de Abril de 2017). Contribución a la detección de objetivos específicos para aplicaciones de vigilancia con realidad aumentada. Doctoral dissertation . Madrid, Madrid, España: ETSIS_Telecomunicacion.

[3] Cruz, H. E. (2016). Efficient forest fire detection index for application in unmanned aerial systems (UASs). Sensors , 893-993.

\section{REFERENCIAS}

[4] Cruz, H. M. (2016). (2016, June). Night Time and Low Visibility Driving Assistance Based on the Application of Colour and Geometrical Features Extraction. En I. I. Cities, Lecture Notes in Computer Science (págs. 118127). Málaga: Springer.

[5] Cruz, H. O. (2017). Precise realtime detection of nonforested areas with UAVs. IEEE Transactions on Geoscience and Remote Sensing, 632-644
[6] Panetta, K. (16 de agosto de 2018). 5 Trends Emerge in the Gartner Hype Cycle for Emerging Technologies, 2018. Recuperado el 24 de septiembre de 2018, de https://www.gartner.com/ smarterwithgartner/5-trends-emergein-gartner-hype-cycle-for-emergingtechnologies-2018/ 